\title{
PLANTS SELECTION FOR DOMESTIC SEWAGE TREATMENT IN JILIN CITY
}

\author{
Jizhong $Q i^{*}$ \\ Yan Zhao \\ Forestry College of Beihua University, P. R. China
}

\begin{abstract}
This experiment studied the removal efficiency of domestic sewage by testing 19 species of wetland plants along the Songhua River in Jilin City. It is shown that plants can purify domestic sewage, and the ability of removal efficiency is varied on different species. Rumex patientia var.callosus is the best in removal efficiency of TN(29.14\%);Coleus blumei Benth. could effectively reduce output of TP and COD, the best removal efficiencies for TP is $58.04 \%$, COD is $95.18 \%$; Oenanthe javanica(Blume) DC. has better efficiency to increase DO in sewage, and the maximum increase is 262.22\%;Rumex.patientia var.callosus,Alisma orientale(Sam.)Juz.,Oenanthe javanica(Blume) DC.,Erigeron annuus (L. ) Pers. may be used as plants for the construction of sewage treatment landscape, because these plants have better comprehensive capacity for domestic sewage treatment.

Water pollution has become a general and worldwide problem today. The application of wetland plants for waste water treatment features low cost,easiness to manage, high efficiency and etc.(Han Xiaoyuan etc.,2005;Li linfeng etc.,2006;Zhang Honggang,Hong Jianming,2006). Wetland plants can not only absorb nitrogen and phosphorus directly but also remove the heavy metals and organics in the waste water. (Xu Weiwei etc.,2005;Cheng Wei etc.,2005;C.C.Tanner,2001;Tang Shirong,2006;SAMAKEMoussa,2003;L.K.Mitchell,A.D.

Karathanasis,1995).According to current research reports,Phragmitas communis Trin, Juncus effuses Linn, Rush, and Iris japonica Thunb, Buttery Swordflag can efficiently remove nitrogen and phosphorus from the sewage (Deng Futang etc.,2005; Yuan Donghai etc.,2004;A. Fey,G. Benckiser,J.C.G. Ottow,1999).The constructed wetlands consisting of varied plants,such as Phragmitas communis Trin,Canna indica Linn. or Acorus gramineus Soland,Grassleaved Sweetflag,are effective in eliminating chemical oxygen demand("COD") in the waste water(Zhao Jiangang etc.,2006;Zhong Yushu etc.,2006; Yuan Donghai etc.,2004). Therefore wetlands can be used to reduce water pollution(Weng Meiya etc.,2005;Liu Zilian etc.,2005;Robert M. Seams,1995).So far most current studies have been developed in the South China with few in the Northeast area of China.

19 kinds of wetland plants growing along the Songhua River is selected in Jinlin City. We analyze and decide which plants are suitable for plant landscape application due to their better urban sewage treatment functions.
\end{abstract}

\section{KEY WORDS}

Domestic sewage, purification, plant, selection

https://doi.org/10.15626/Eco-Tech.2010.091 


\section{MATERIALS AND METHODS}

\subsection{Materials}

\subsubsection{Water sample}

Sample water was collected from the domestic sewage outfall under the Linjiangmen Bridge of Songhua River in Jilin City at 6:00 A.M. Afterwards we poured the various kinds of plants with such sample water (or "Input Water")to compare and analyze their experimental indicators simultaneously.

\subsubsection{Plants}

19 species of plants healthily growing nearby the sewage outlets along Songhua River is selected as the study objects (Table 1), based on their adaptability to environment, strength to resist pollution substances and the capability of pest resistance.

Tab.1 Plants Analyzed in the Experiments

\begin{tabular}{|c|c|c|}
\hline No. & Chinese & Latin Name \\
\hline 1 & 泽泻 & Alisma orientale (Sam.)Juz. \\
\hline 2 & 和尚菜 & Adenocaulon himalaicum Maxim. \\
\hline 3 & 圧绿㢣 & Chenopodium glaucum L. \\
\hline 4 & 洋铁酸模 & Rumex patientia var.callosus F.Schmidt \\
\hline 5 & 桃叶蓼 & Polygonum persicaria L. \\
\hline 6 & 黑心菊 & Rudbeckia hybrida \\
\hline 7 & 戟叶蓼 & Polygonum thunbergii Sieb.et Zucc. \\
\hline 8 & 马萄 & Iris lactea var.chinensis Thunb \\
\hline 9 & 紫楞 & Hosta ventricosa Stearn \\
\hline 10 & 彩叶草 & Coleus blumei Benth. \\
\hline 11 & 艾蒿 & Artemisia argyi Levl.et Vant. \\
\hline 12 & 白三叶 & Txifolium repens L. \\
\hline 13 & 山莴苣 & Lactuca indica L. \\
\hline 14 & 美汉草 & Meehania urticifolia (Miq.)Makino \\
\hline 15 & 毛莨 & Ranunculus japonicus Thunb. \\
\hline 16 & 连钱草 & Glechoma hederacea L.var.longituba Nakai. \\
\hline 17 & 一年蓬 & Erigeron annuus (L.) Pers. \\
\hline 18 & 水芹 & Oenanthe javanica(Blume) DC. \\
\hline 19 & 鸭跖草 & Commelina communis L. \\
\hline
\end{tabular}




\subsection{Analysis Methods}

\subsubsection{Devices}

Flowerpots are used to contain and grow the plants, each of which has a top diameter of 37 centimeters("cm"), a bottom diameter of $25 \mathrm{~cm}$, a height of $45 \mathrm{~cm}$ and the $22 \mathrm{~L}$ volume. We placed a $10 \mathrm{~cm}$-thick layer of cobblestones each measuring $3-8 \mathrm{~cm}$ in diameter as water filter, topped with a piece of plastic window screening to prevent the sands from filling the spaces among the cobblestones. On top of the plastic window screening, there's a layer of river sand and soil with a $25 \mathrm{~cm}$ thickness to grow the plants("Growing Base"). We inserted a plastic pipe with a $0.5 \mathrm{~cm}$ diameter into the water filter so as to output the water samples (“Output Water”) for analyzing.

\subsubsection{Analyzing Procedures.}

The plants are planted on the Growing Base in the earlier spring. Each kinds of plants have three groups of repeated experimental data compared with three groups of original data(Growing Base Without Plant). The objects are poured the Input Water once in 10 days. After staying in the flowerpots for 10 days, the Output Water is siphoned and immediately analyze the total nitrogen (TN), total phosphorus (TP), chemical oxygen demand (COD), dissolved oxygen (DO) contained by the Output Water in the lab.

\subsubsection{Analysis Method}

According to the Analysis Methods for Water and Waste Water published by State Administration of Environmental Protection, chemical analysis was carried out following the methods:

determination of TN: Alkaline potassium persulfate digestion-UV spectrophotometry method;

determination of TP: Ammonium molybdate spectrophotometric method;

determination of COD: $\quad$ Potassium dichromate method;

determination of DO: $\quad$ Iodimetry.

\section{RESULTS AND ANALYSIS}

The water quality was measured on July 26, August 6, August 15, and August 26 during the experiment period. Removing rates (\%) of TN, TP, COD and increasing rate of DO in the water were used for the study. Equation for removing rates of TN, TP, and COD were:

Removing rate $=($ control concentration - measured concentration $) /$ influent water sample concentration $\times 100 \%$

Equation for increasing rate of DO: 
Increasing rate $=$ (measured concentration - control concentration)/influent water sample concentration $\times 100 \%$

\subsection{Results produced by different plants for removing TN in domestic sewage}

Table 2 shows the TN removing rates in different periods during the experiment. The rates are calculated according to $\mathrm{TN}$ concentration before and after the treatment by plants.

Tab.2 TN Removing Results of Different Plants

\begin{tabular}{|c|c|c|c|c|c|}
\hline \multirow{2}{*}{$\begin{array}{l}\text { No } \\
\cdot\end{array}$} & \multirow{2}{*}{ Plants } & \multicolumn{4}{|c|}{ Removal ( \% ) } \\
\hline & & July 26 & Aug.6 & Aug.15 & Aug.26 \\
\hline 1 & Alisma orientale (Sam.)Juz. & 28.5639 & 2.6001 & 1.4230 & 0.4184 \\
\hline 2 & $\begin{array}{l}\text { Adenocaulon himalaicum } \\
\text { Maxim. }\end{array}$ & 1.8526 & 4.4303 & 1.3063 & 1.3689 \\
\hline 3 & Chenopodium glaucum L. & 0.4837 & 0.3761 & 1.3489 & 1.4176 \\
\hline 4 & Rumex patientia var.callosus & 29.1353 & 17.9879 & 0.7561 & 1.6357 \\
\hline 5 & $\begin{array}{l}\text { F.Schmidt } \\
\text { Polygonum persicaria L. }\end{array}$ & 27.2340 & 19.8897 & 1.0688 & 2.4978 \\
\hline 6 & Rudbeckia hybrida & 18.1210 & 3.2120 & 12.4213 & 1.1343 \\
\hline 7 & $\begin{array}{l}\text { Polygonum thunbergii Sieb.et } \\
\text { Zucc. }\end{array}$ & 24.2269 & 8.1949 & 0.8178 & 6.3522 \\
\hline 8 & Iris lactea var.chinensis Thunb & 25.5212 & 21.0061 & 1.4332 & 3.8069 \\
\hline 9 & Hosta ventricosa Stearn & 23.6735 & 10.6034 & 1.2056 & 2.7811 \\
\hline 10 & Coleus blumei Benth. & 19.0982 & 9.6979 & 0.8212 & 5.4269 \\
\hline 11 & Artemisia argyi Levl.et Vant. & 22.5843 & 16.1651 & 1.1470 & 1.5483 \\
\hline 12 & Txifolium repens L. & 2.7252 & 10.2567 & 1.2664 & 2.9374 \\
\hline 13 & Lactuca indica L. & 21.0714 & 4.7546 & 0.8805 & 3.4309 \\
\hline 14 & $\begin{array}{ll}\text { Meehania } & \text { urticifolia } \\
\text { (Miq.)Makino } & \end{array}$ & 25.8014 & 11.6119 & 1.3482 & 2.8499 \\
\hline 15 & Ranunculus japonicus Thunb. & 25.2410 & 1.8009 & 0.6727 & 1.0583 \\
\hline 16 & $\begin{array}{l}\text { Glechoma hederacea } \\
\text { L.var.longituba Nakai. }\end{array}$ & 25.6746 & 10.7548 & 1.6004 & 0.5741 \\
\hline
\end{tabular}




\begin{tabular}{llllll}
\hline 17 & Erigeron annuus (L.) Pers. & 23.0211 & 12.3546 & 11.1413 & 2.5215 \\
18 & Oenanthe javanica(Blume) DC. & 28.3632 & 14.8089 & 0.5746 & 1.0594 \\
19 & Commelina communis L. & 28.2097 & 12.8398 & 0.6734 & 1.8309 \\
\hline
\end{tabular}

These rates show that most of the plants are at the highest performance level for removing TN around July 26. The performance follows a downward trend afterwards and picks up slightly on August 26. In particular, Rumex patientia var.callosus F.Schmidt is most effective with removing rate up to $29.13 \%$. Those less effective plants include Adenocaulon himalaicum Maxim., Txifolium repens L., and Chenopodium glaucum L. with removing rate less than $10 \%$.

Multiple comparison of TN removing rate of different plants in their most effective period shows that Rumex patientia var.callosus F.Schmidt, Alisma orientale (Sam.)Juz., Oenanthe javanica(Blume) DC., and Commelina communis L. are more effective, Polygonum persicaria L., Lactuca indica L., Ranunculus japonicus Thunb., Polygonum thunbergii Sieb.et Zucc., and Meehania urticifolia (Miq.)Makino are moderate, while others are less effective. Figure 1 shows the curves of purifying process of those plants with higher performance.

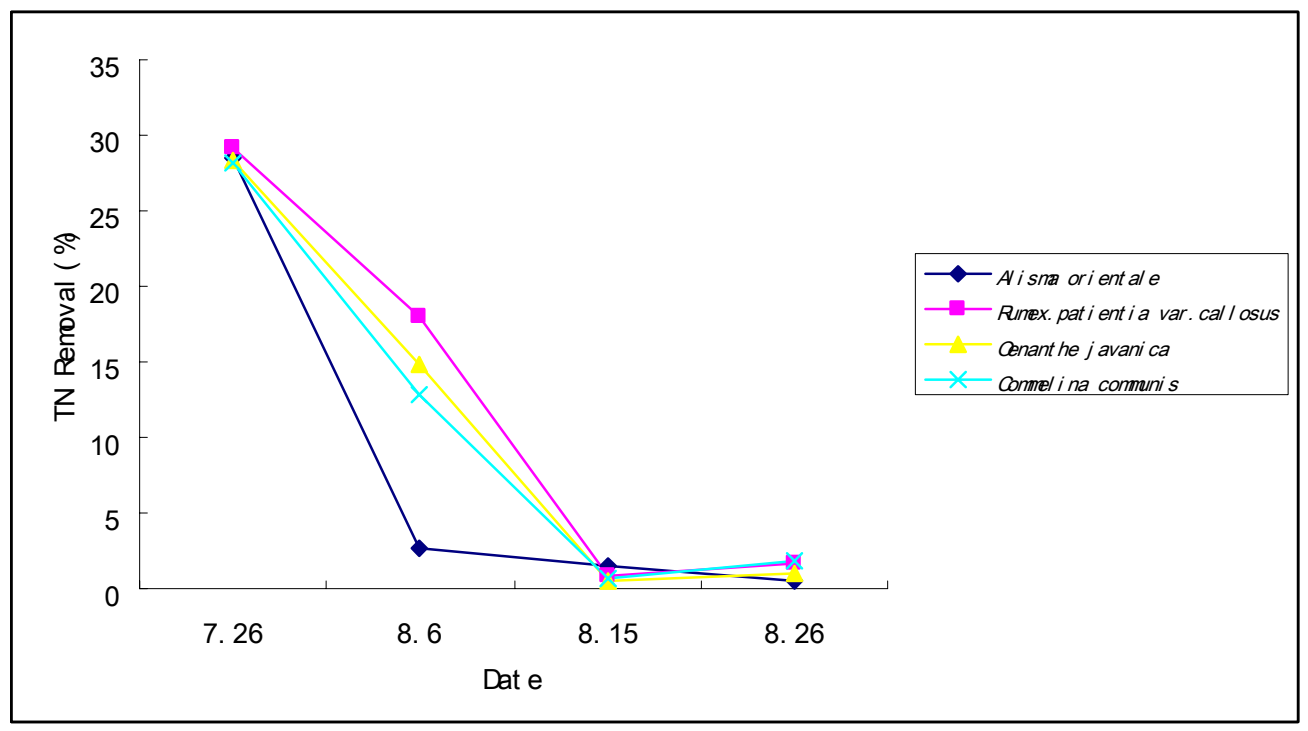

Fig. 1 TN Removing Results of Plants with Higher Performance

\subsection{Results produced by different plants for removing TP in domestic sewage}

Table 3 shows the TP removing rates in different periods by different plants. 
Tab.3 TP Removing Results of Different Plants

No. Plants

Removal ( \% )

\begin{tabular}{|c|c|c|c|c|c|}
\hline & & July 26 & Aug. 6 & Aug.15 & Aug.26 \\
\hline 1 & Alisma orientale (Sam.)Juz. & 40.3134 & 1.9822 & 43.7959 & 3.7326 \\
\hline 2 & $\begin{array}{l}\text { Adenocaulon himalaicum } \\
\text { Maxim. }\end{array}$ & 42.4933 & 21.8052 & 15.4448 & 5.4291 \\
\hline 3 & Chenopodium glaucum L. & 13.3381 & 0.6738 & 9.2284 & 1.4945 \\
\hline 4 & $\begin{array}{l}\text { Rumex patientia var.callosus } \\
\text { F.Schmidt }\end{array}$ & 43.3711 & 3.1064 & 38.5123 & 5.5836 \\
\hline 5 & Polygonum persicaria L. & 44.6646 & 3.3443 & 8.2651 & 4.2169 \\
\hline 6 & Rudbeckia hybrida & 43.1836 & 19.5471 & 12.6756 & 2.1029 \\
\hline 7 & $\begin{array}{l}\text { Polygonum thunbergii Sieb.et } \\
\text { Zucc. }\end{array}$ & 41.8821 & 11.4086 & 45.0056 & 3.8312 \\
\hline 8 & $\begin{array}{l}\text { Iris lactea var.chinensis } \\
\text { Thunb }\end{array}$ & 39.2991 & 3.6087 & 26.2344 & 2.3687 \\
\hline 9 & Hosta ventricosa Stearn & 33.7963 & 3.7965 & 10.4939 & 4.9982 \\
\hline 10 & Coleus blumei Benth. & 45.0132 & 25.8707 & 58.0432 & 3.5974 \\
\hline 11 & Artemisia argyi Levl.et Vant. & 11.2880 & 3.4477 & 8.9707 & 3.3195 \\
\hline 12 & Txifolium repens L. & 0.6357 & 2.7328 & 4.5074 & 3.3303 \\
\hline 13 & Lactuca indica L. & 5.7970 & 6.3640 & 2.4226 & 4.8201 \\
\hline 14 & $\begin{array}{ll}\text { Meehania } & \text { urticifolia } \\
\text { (Miq.)Makino } & \end{array}$ & 16.6732 & 3.4434 & 2.4202 & 2.7716 \\
\hline 15 & Ranunculus japonicus Thunb. & 18.8343 & 4.7228 & 9.5769 & 1.9540 \\
\hline 16 & Glechoma hederacea & 14.2385 & 3.0912 & 1.0901 & 3.2373 \\
\hline 17 & $\begin{array}{l}\text { L.var.longituba Nakai. } \\
\text { Erigeron annuus (L.) Pers. }\end{array}$ & 44.7550 & 16.4736 & 0.5125 & 3.2561 \\
\hline 18 & $\begin{array}{l}\text { Oenanthe javanica(Blume) } \\
\text { DC. }\end{array}$ & 14.6099 & 3.5058 & 13.7460 & 10.5836 \\
\hline 19 & Commelina communis L. & 28.5625 & 8.7747 & 29.4353 & 6.8614 \\
\hline
\end{tabular}

Most of the plants are at higher performance level for removing TP around July 26. The highest removing rate of Coleus blumei Benth. is 58.04\%. The rate on August 6 decreases while the rate on August 16 increases before the decrease for the second time. 
Txifolium repens L., Lactuca indica L., and Glechoma hederacea L.var.longituba Nakai. are always at lower performance for removing TP.

Multiple comparison shows that Coleus blumei Benth., Polygonum thunbergii Sieb.et Zucc., Erigeron annuus (L.) Pers., Polygonum persicaria L., Alisma orientale (Sam.)Juz., Rudbeckia hybrida, Adenocaulon himalaicum Maxim., Iris lactea var.chinensis Thunb, and Rumex patientia var.callosus F.Schmidt are more effective, Hosta ventricosa Stearn, Commelina communis L., Ranunculus japonicus Thunb., and Artemisia argyi Levl.et Vant. are moderate, while Oenanthe javanica(Blume) DC., Meehania urticifolia (Miq.)Makino, Chenopodium glaucum L., Glechoma hederacea L.var.longituba Nakai., Lactuca indica L., and Txifolium repens L. are less effective. Figure 2 shows the purifying process of those plants with higher performance.

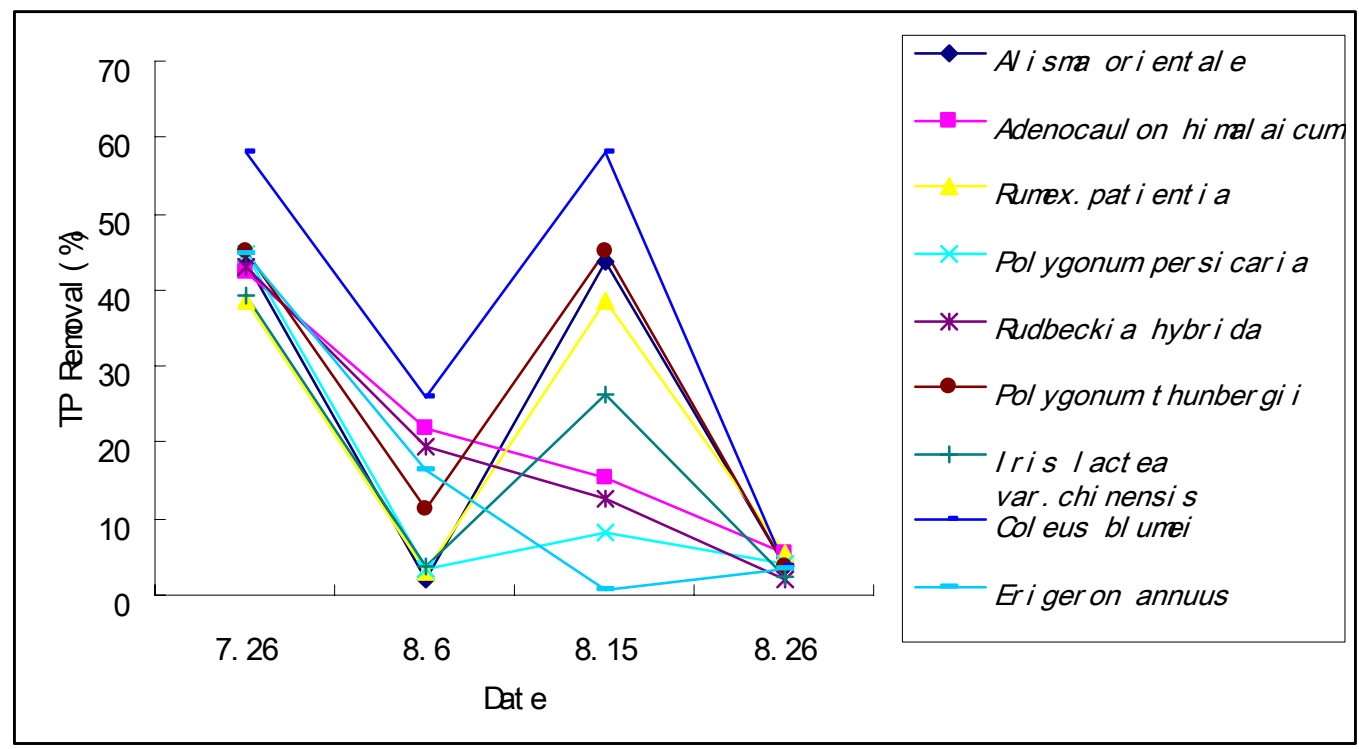

Fig .2 TP Removing Results of Plants with Higher Performance

\subsection{Results produced by different plants for removing COD in domestic sewage}

Table 4 shows the COD removing rates by different plants. 
Tab.4 COD Removing Results of Different Plants

\begin{tabular}{|c|c|c|c|c|c|}
\hline \multirow{2}{*}{ No. } & \multirow{2}{*}{ Plants } & \multicolumn{4}{|c|}{ Removal ( \% ) } \\
\hline & & July 26 & Aug.6 & Aug.15 & Aug.26 \\
\hline 1 & Alisma orientale(Sam.)Juz. & 43.2031 & 71.2935 & 43.7959 & 3.7326 \\
\hline 2 & $\begin{array}{l}\text { Adenocaulon himalaicum } \\
\text { Maxim. }\end{array}$ & 44.1445 & 91.1165 & 2.0039 & 5.4292 \\
\hline 3 & Chenopodium glaucum L. & 14.7141 & 14.7140 & 9.2284 & 1.4945 \\
\hline 4 & Rumex.patientia var.callosus & 46.7437 & 72.4176 & 11.6306 & 5.5836 \\
\hline 5 & $\begin{array}{l}\text { F.Schmidt } \\
\text { Polygonum persicaria L. }\end{array}$ & 46.0406 & 72.6556 & 8.2651 & 4.2169 \\
\hline 6 & Rudbeckia hybrida & 46.5823 & 88.8583 & 12.6756 & 2.5103 \\
\hline 7 & Polygonum thunbergii & 44.0837 & 80.7198 & 45.0056 & 3.8312 \\
\hline 8 & $\begin{array}{l}\text { Sieb.et Zucc. } \\
\text { Iris lactea var.chinensis }\end{array}$ & 45.3534 & 72.9199 & 18.1698 & 2.3686 \\
\hline 9 & $\begin{array}{l}\text { Thunb } \\
\text { Hosta ventricosa Stearn }\end{array}$ & 45.6298 & 73.1077 & 10.4939 & 4.9982 \\
\hline 10 & Coleus blumei Benth. & 46.3891 & 95.1819 & 58.0432 & 3.5974 \\
\hline 11 & Artemisia argyi Levl.et & 14.0399 & 14.0399 & 8.9706 & 3.3194 \\
\hline 12 & $\begin{array}{l}\text { Vant. } \\
\text { Txifolium repens L. }\end{array}$ & 0.6357 & 4.5074 & 4.5074 & 3.3303 \\
\hline 13 & Lactuca indica L. & 5.7970 & 17.0379 & 2.4226 & 4.8201 \\
\hline 14 & Meehania & 16.6731 & 16.6731 & 2.4202 & 2.7716 \\
\hline 15 & $\begin{array}{l}\text { (Miq.)Makino } \\
\text { Ranunculus } \\
\text { Thunb. }\end{array}$ & 18.8343 & 29.0136 & 9.5769 & 1.9540 \\
\hline 16 & Glechoma hederacea & 22.4944 & 22.4944 & 1.0900 & 3.2373 \\
\hline 17 & $\begin{array}{l}\text { L.var.longituba Nakai. } \\
\text { Erigeron annuus (L.) Pers. }\end{array}$ & 46.1310 & 71.8948 & 0.5124 & 3.2560 \\
\hline 18 & $\begin{array}{l}\text { Oenanthe javanica(Blume) } \\
\text { DC. }\end{array}$ & 17.3618 & 17.3618 & 13.7460 & 10.5836 \\
\hline 19 & Commelina communis L. & 31.5897 & 78.0860 & 1.8681 & 6.8614 \\
\hline
\end{tabular}

In the experiment period, COD removing rate indicates an upward trend followed by a downward trend. Rumex.patientia var.callosus F.Schmidt has the highest COD removing rate of up to $95.18 \%$ while Txifolium repens $\mathrm{L}$. has the least rate of less than $5 \%$. 
Comparison by least significant ranges shows that Erigeron annuus (L.) Pers., Rumex patientia var.callosus F.Schmidt, Rudbeckia hybrida, Hosta ventricosa Stearn, Polygonum persicaria L., and Coleus blumei Benth. are more effective, Oenanthe javanica(Blume) DC., Ranunculus japonicus Thunb., Lactuca indica L., Iris lactea var.chinensis Thunb, Polygonum thunbergii Sieb.et Zucc., Glechoma hederacea L.var.longituba Nakai., Adenocaulon himalaicum Maxim., and Alisma orientale (Sam.)Juz. are moderate, while Commelina communis L., Txifolium repens L., Chenopodium glaucum L., Meehania urticifolia (Miq.)Makino, and Artemisia argyi Levl.et Vant. are less effective. Figure 3 shows the curve of purifying process of those plants with higher performance.

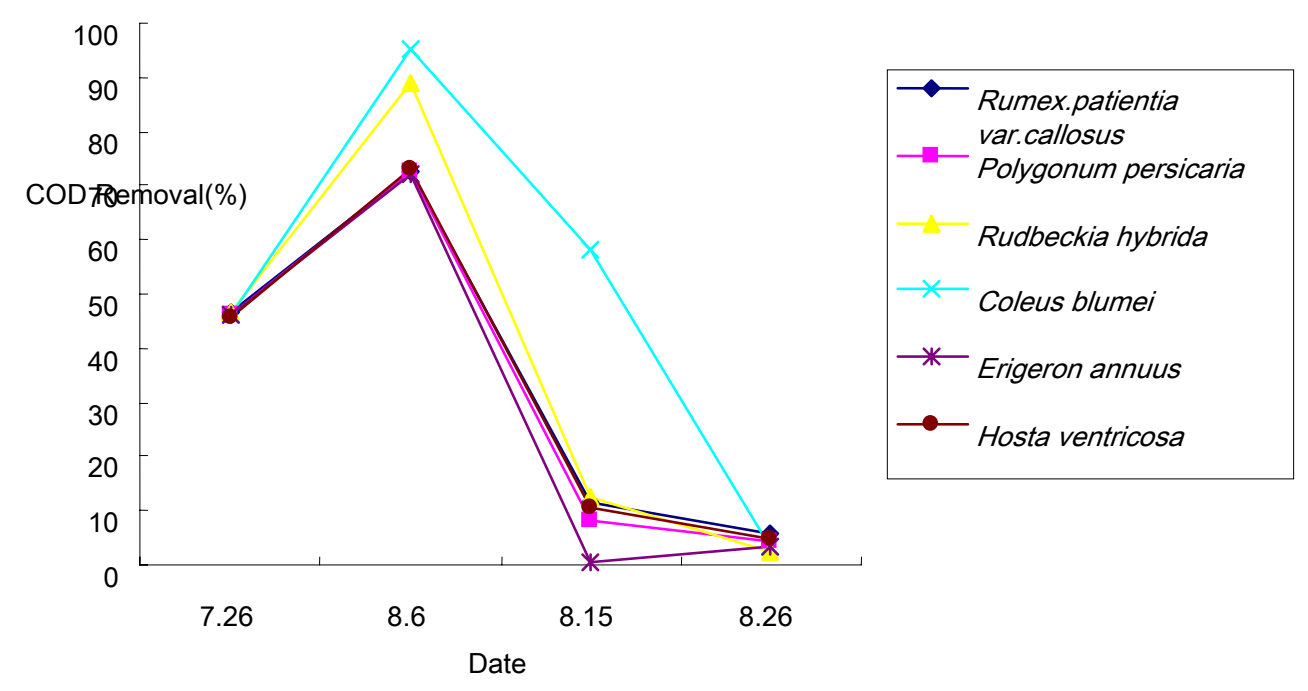

Fig.3 COD Removing Results of Plants with Higher Performance

\subsection{Results produced by different plants for removing DO in domestic sewage}

DO increasing results of different plants are shown in Table 5. 
Tab.5 DO Increasing Results of Different Plants

\begin{tabular}{|c|c|c|c|c|c|}
\hline \multirow{2}{*}{ No. } & \multirow{2}{*}{ Plants } & \multicolumn{4}{|c|}{ Increase ( \% ) } \\
\hline & & July 26 & Aug.6 & Aug.15 & Aug.26 \\
\hline 1 & Alisma orientale(Sam.)Juz. & 52.3809 & 18.9393 & 60 & 6.6326 \\
\hline 2 & Adenocaulon himalaicum & 40.4761 & 46.2121 & 108.8889 & 10.3741 \\
\hline 3 & Chenopodium glaucum L. & 7.1428 & 44.6969 & 44.6969 & 10.5102 \\
\hline 4 & Rumex.patientia var.callosus & 107.1428 & 108.3333 & 108.3333 & 24.5578 \\
\hline 5 & $\begin{array}{l}\text { F.Schmidt } \\
\text { Polygonum persicaria L. }\end{array}$ & 73.8095 & 61.3636 & 135.5556 & 19.5238 \\
\hline 6 & Rudbeckia hybrida & 21.4285 & 29.5454 & 106.6667 & 11.6667 \\
\hline 7 & Polygonum thunbergii Sieb.et & 92.8571 & 15.9091 & 95.5556 & 11.7346 \\
\hline 8 & $\begin{array}{l}\text { Zucc. } \\
\text { Iris lactea var.chinensis }\end{array}$ & 114.2857 & 97.7273 & 114.2857 & 15.1361 \\
\hline 9 & $\begin{array}{l}\text { Thunb } \\
\text { Hosta ventricosa Stearn }\end{array}$ & 95.2381 & 67.4242 & 95.2381 & 15.7823 \\
\hline 10 & Coleus blumei Benth. & 161.9047 & 131.0606 & 247.7778 & 10.1361 \\
\hline 11 & Artemisia argyi Levl.et Vant. & 52.3809 & 43.1818 & 68.8889 & 15.0340 \\
\hline 12 & Txifolium repens L. & 111.9047 & 99.2424 & 111.9047 & 22.7891 \\
\hline 13 & Lactuca indica L. & 14.2857 & 79.5454 & 57.7778 & 20.2721 \\
\hline 14 & Meehania & 83.3333 & 115.9091 & 131.1111 & 16.9728 \\
\hline 15 & $\begin{array}{l}\text { (Miq.)Makino } \\
\text { Ranunculus japonicus Thunb. }\end{array}$ & 128.5714 & 81.0606 & 128.5714 & 14.3877 \\
\hline 16 & Glechoma hederacea & 121.4285 & 56.8181 & 121.4285 & 10.8503 \\
\hline 17 & $\begin{array}{l}\text { L.var.longituba Nakai. } \\
\text { Erigeron annuus (L.) Pers. }\end{array}$ & 154.7619 & 90.1515 & 154.7619 & 11.4626 \\
\hline 18 & $\begin{array}{l}\text { Oenanthe javanica(Blume) } \\
\text { DC. }\end{array}$ & 21.4285 & 40.1515 & 262.2222 & 15.4761 \\
\hline 19 & Commelina communis L. & 90.4761 & 65.9091 & 153.3333 & 3.7415 \\
\hline
\end{tabular}

During the initial period of the experiment, DO Increasing rates of most plants decrease before a pick up. The lowest point is on August 26. Oenanthe javanica(Blume) DC. has the highest rate of increasing DO of up to $262.22 \%$ while Chenopodium glaucum L. is less effective, with a rate always less than $50 \%$. 
Multiple comparison shows that Oenanthe javanica(Blume) DC., Coleus blumei Benth., Erigeron annuus (L.) Pers., and Commelina communis L. are more effective, Polygonum persicaria L., Meehania urticifolia (Miq.)Makino, and Ranunculus japonicus Thunb. are moderate, while Glechoma hederacea L.var.longituba Nakai., Iris lactea var.chinensis Thunb, Txifolium repens L., Adenocaulon himalaicum Maxim., Rumex patientia var.callosus F.Schmidt, Rudbeckia hybrida, Polygonum thunbergii Sieb.et Zucc., Hosta ventricosa Stearn, Artemisia argyi Levl.et Vant., Alisma orientale (Sam.)Juz., and Lactuca indica L. are less effective. Figure 4 shows the curve of purifying process of those plants with higher performance.

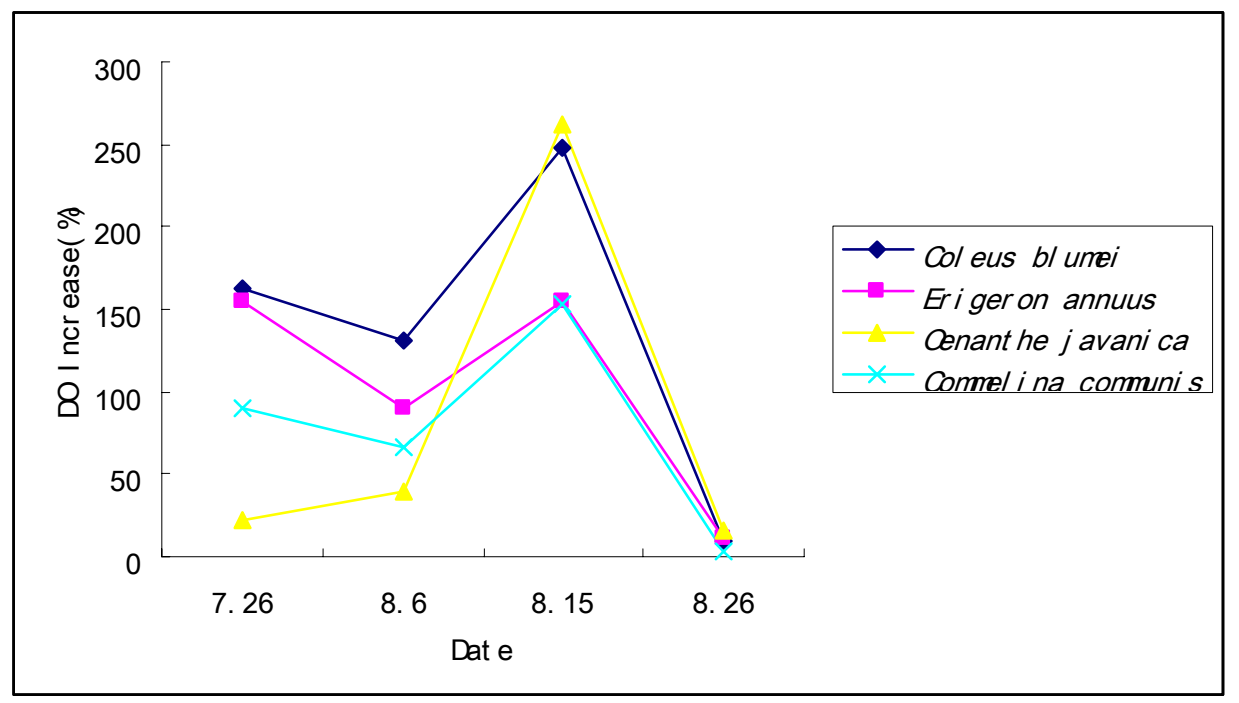

Fig.4 DO Increasing Results of Plants with Higher Performance

\subsection{Comparison of comprehensive results of different plants}

Weight and comprehensive scores (A) of the four indicators are determined according to the assessment method proposed by experts (Wang Yi, 2005) in their study on water purification system for wetland.

$\mathrm{A}=\mathrm{FTP} \times \omega \mathrm{TP}+\mathrm{FTN} \times \omega \mathrm{TN}+\mathrm{FCOD} \times \omega \mathrm{COD}+\mathrm{FDO} \times \omega \mathrm{DO}$

$\omega \mathrm{TP}=\mathrm{PTP} / \mathrm{P} ; \omega \mathrm{TN}=\mathrm{PTN} / \mathrm{P} ; \omega \mathrm{COD}=\mathrm{PCOD} / \mathrm{P} ; \omega \mathrm{DO}=\mathrm{PDO} / \mathrm{P}$

$\mathrm{P}=\mathrm{PTP}+\mathrm{PTN}+\mathrm{PCOD}+\mathrm{PDO}$

$\mathrm{PTP}=\mathrm{TPi} / \mathrm{TP}$; $\mathrm{PTN}=\mathrm{TNi} / \mathrm{TNo} ; \mathrm{PCOD}=\mathrm{CODi} / \mathrm{CODo} ; \mathrm{P} \quad$ (DO saturated $-\mathrm{DOi}) /(\mathrm{DO}$ saturated - DOo);

$\omega \mathrm{TP}, \omega \mathrm{TN}, \omega \mathrm{COD}, \omega \mathrm{DO}$ - weight of TP, TN, COD, DO;

TPi, TPo - measured value and benchmark of TP; 
TNi, TNo - measured value and benchmark of TN;

CODi, CODo - measured value and benchmark of CO;

DO saturated, DOi, DOo - Saturated dissolved oxygen concentration under water temperature of measurement as well as the measured value and benchmark of DO;

The benchmarks are provided in Environmental Quality Standard of Surface Water (GB3838-2002) as water quality $(\mathrm{TNo} \leq 1.0 \mathrm{mg} / \mathrm{L}, \mathrm{TPo} \leq 0.2 \mathrm{mg} / \mathrm{L}, \mathrm{CODo} \leq 20 \mathrm{mg} / \mathrm{L}$, $\mathrm{DOo} \geq 5 \mathrm{mg} / \mathrm{L})$.

Removing rate (\%) is scored according to the following rules in Table 6 by using the experimental results.

Tab.6 score criterion (F)

\begin{tabular}{|c|c|c|c|c|c|c|}
\hline $\mathbf{F}$ & 5 & 4 & 3 & & 2 & 1 \\
\hline $\mathrm{TN}$ & Removal $>27$ & $\begin{array}{l}25<\text { Removal } \\
<27\end{array}$ & $\begin{array}{l}20< \\
<27\end{array}$ & Removal & $\begin{array}{l}10<\text { Removal } \\
<20\end{array}$ & Removal $<10$ \\
\hline TP & $\begin{array}{l}\text { Removal } \\
>40\end{array}$ & $\begin{array}{l}30<\text { Removal } \\
<40\end{array}$ & $\begin{array}{l}20< \\
<30\end{array}$ & Removal & $\begin{array}{l}10<\text { Removal } \\
<20\end{array}$ & Removal $<10$ \\
\hline COD & $\begin{array}{l}\text { Removal } \\
>50\end{array}$ & $\begin{array}{l}40<\text { Removal } \\
<50\end{array}$ & $\begin{array}{l}30< \\
<40\end{array}$ & Removal & $\begin{array}{l}20<\text { Removal } \\
<30\end{array}$ & $\begin{array}{l}10<\text { Removal } \\
20\end{array}$ \\
\hline DO & Increase $>200$ & $\begin{array}{l}150<\text { Increase } \\
<200\end{array}$ & $\begin{array}{l}125< \\
<150\end{array}$ & Increase & $\begin{array}{l}100<\text { Increase } \\
<125\end{array}$ & $\begin{array}{l}\text { Increase } \\
<100\end{array}$ \\
\hline
\end{tabular}

Table 7 shows the comprehensive scores calculated according to the above equations. 
Tab.7 The sort order of total score

\begin{tabular}{|c|c|c|c|c|c|c|}
\hline \multirow{2}{*}{ No. } & \multirow{2}{*}{ Plants } & \multicolumn{4}{|c|}{ Analysis of indicators } & \multirow{2}{*}{ score } \\
\hline & & $\mathbf{T N}$ & $\mathbf{T P}$ & COD & DO & \\
\hline 1 & $\begin{array}{l}\text { Rumex patientia var.callosus } \\
\text { F.Schmidt }\end{array}$ & 1.3579 & 0.5235 & 3.6126 & 0.1336 & 5.6276 \\
\hline 2 & Alisma orientale (Sam.)Juz. & 2.2103 & 1.4457 & 1.7459 & 0.1134 & 5.5153 \\
\hline 3 & $\begin{array}{l}\text { Oenanthe javanica(Blume) } \\
\text { DC. }\end{array}$ & 1.9338 & 0.1105 & 1.5338 & 1.8914 & 5.4695 \\
\hline 4 & Erigeron annuus (L.) Pers. & 1.3129 & 0.2316 & 3.2968 & 0.4333 & 5.2746 \\
\hline 5 & Polygonum persicaria L. & 1.0595 & 0.1668 & 3.2654 & 0.2609 & 4.7526 \\
\hline 6 & Coleus blumei Benth. & 0.9615 & 1.6879 & 0.3545 & 1.7073 & 4.7112 \\
\hline 7 & Rudbeckia hybrida & 0.7574 & 0.6438 & 2.3762 & 0.4202 & 4.1976 \\
\hline 8 & $\begin{array}{l}\text { Polygonum thunbergii Sieb.et } \\
\text { Zucc. }\end{array}$ & 1.5314 & 1.3839 & 0.8406 & 0.1524 & 3.9083 \\
\hline 9 & $\begin{array}{l}\text { Iris lactea var.chinensis } \\
\text { Thunb }\end{array}$ & 1.4452 & 0.9399 & 1.0325 & 0.0972 & 3.5148 \\
\hline 10 & Ranunculus japonicus Thunb. & 1.6649 & 0.0929 & 1.3692 & 0.1748 & 3.3018 \\
\hline 11 & Commelina communis L. & 1.5929 & 0.2937 & 0.6796 & 0.6943 & 3.2605 \\
\hline 12 & Lactuca indica L. & 1.6087 & 0.0564 & 1.2781 & 0.1239 & 3.0671 \\
\hline 13 & $\begin{array}{ll}\text { Meehania } & \text { urticifolia } \\
\text { (Miq.)Makino } & \end{array}$ & 1.6903 & 0.0513 & 0.5703 & 0.6526 & 2.9645 \\
\hline 14 & $\begin{array}{l}\text { Glechoma hederacea } \\
\text { L.var.longituba Nakai. }\end{array}$ & 0.9984 & 0.0314 & 1.6347 & 0.0711 & 2.7356 \\
\hline 15 & Hosta ventricosa Stearn & 1.0177 & 0.5657 & 0.5695 & 0.0658 & 2.2187 \\
\hline 16 & $\begin{array}{l}\text { Adenocaulon himalaicum } \\
\text { Maxim. }\end{array}$ & 0.0578 & 0.7268 & 0.4582 & 0.421 & 1.6638 \\
\hline 17 & Artemisia argyi Levl.et Vant. & 0.8339 & 0.1594 & 0.5226 & 0.1408 & 1.6567 \\
\hline 18 & Txifolium repens L. & 0.2087 & 0.0619 & 0.7145 & 0.1327 & 1.1178 \\
\hline 19 & Chenopodium glaucum L. & 0.0119 & 0.0819 & 0.7239 & 0.0469 & 0.8646 \\
\hline
\end{tabular}


Performance of different plants is ranked as Rumex patientia var.callosus F.Schmidt $>$ Alisma orientale (Sam.)Juz.> Oenanthe javanica(Blume) DC. > Erigeron annuus (L.) Pers. > Polygonum persicaria L. > Coleus blumei Benth.> Rudbeckia hybrida > Polygonum thunbergii Sieb.et Zucc.> Iris lactea var.chinensis Thunb $>$ Ranunculus japonicus Thunb.> Commelina communis L.> Lactuca indica L.> Meehania urticifolia (Miq.)Makino > Glechoma hederacea L.var.longituba > Hosta ventricosa Stearn > Adenocaulon himalaicum Maxim.> Artemisia argyi Levl.et Vant.> Txifolium repens L. $>$ Chenopodium glaucum L.

\section{CONCLUSION}

\subsection{Different purifying plants for treating domestic sewage have different performance levels.}

3.2 Among the subjects, those scored more than 5 points include Rumex patientia var.callosus F.Schmidt, Alisma orientale (Sam.)Juz., Oenanthe javanica(Blume) DC. and Erigeron annuus (L.) Pers.; Polygonum persicaria L., Coleus blumei Benth., and Rudbeckia hybrida are between 4-5 points; Polygonum thunbergii Sieb.et Zucc., Iris lactea var.chinensis Thunb, Ranunculus japonicus Thunb., Commelina communis L., and Lactuca indica L. are 3 - 4 points; Meehania urticifolia (Miq.)Makino, Glechoma hederacea L.var.longituba Nakai., and Hosta ventricosa Stearn are 2 - 3 points, Adenocaulon himalaicum Maxim., Artemisia argyi Levl.et Vant.and Txifolium repens L. are $1-2$ points, and Chenopodium glaucum L. is less than 1 point.

\section{DISCUSSION}

4.1 In construction landscape for water purification, Rumex patientia var.callosus F.Schmidt, Alisma orientale (Sam.)Juz., Oenanthe javanica(Blume) DC., and Commelina communis L. may be used in sewage with higher concentration of nitrogen; Coleus blumei Benth., Polygonum thunbergii Sieb.et Zucc., Erigeron annuus (L.) Pers., Polygonum persicaria L., Alisma orientale (Sam.)Juz., Rudbeckia hybrida, Adenocaulon himalaicum Maxim., Iris lactea var.chinensis Thunb, and Rumex patientia var.callosus F.Schmidt may be used in sewage with higher concentration of phosphorous; and Erigeron annuus (L.) Pers., Rumex patientia var.callosus F.Schmidt, Rudbeckia hybrida, Hosta ventricosa Stearn, Polygonum persicaria L., and Coleus blumei Benth. may be used in sewage with higher concentration of organic matters. Domestic sewage may be treated by combining plants with higher scores.

4.2 In the experiment, Rudbeckia hybrida demonstrates rather unique properties in removing TN and TP. TN removing performance had a pick up substantially during the experiment. TP removing performance is relatively satisfactory with a slowly downward trend. Adenocaulon himalaicum Maxim. and Erigeron annuus (L.) Pers. also showes a slightly downward trend in removing of TP. Because demand of nitrogen and phosphorous in vegetative and reproductive growth is not the same, longer blooming period of Rudbeckia hybrida and continuous flowering of Adenocaulon himalaicum Maxim. and Erigeron annuus (L.) Pers.may be related to the performance of the plants. 
In order to optimize the performance of multi-objective landscape for water purification, the plants shall be selected based on their TN and TP removing performances, types and ingredients of pollutants removed by plants in vegetative and reproductive growth, as well as the length of phonological periods of related plants.

4.3 COD removing rate is at its highest level on July 26 while DO increasing performance is decreasing. This is because most of organic matters in the sewage are removed through decomposition by microorganism on the plants and surface of substrate. DO consumption of microorganism is at the highest level when COD removing performance is the best. Because decreasing concentration of organic matters also reduces DO consumption, DO concentration picks up gradually when restoring rate of DO is higher than the rate of consumption (Yi Jun etc. 2007; Zhao Jian etc. 2007). It follows that the COD removing and DO increasing process involves not only the plants, but also microorganism and substrate. In the construction of purifying landscape, the plants shall be cultivated for a period to stabilize the performance of the plants, microorganism, and substrate for optimizing the results of organic matters removal.

\section{REFERENCES}

[1] Han-Xiaoyuan,Bi-Jisheng,Song-Zhiwen.2005.Application and advance of aquatic plants in water pollution control[J]. Qingdao Technological University,26(6):88-90

[2] Li-linfeng,Nian-yuegang,Jiang-Gaoming.2006.Macrophytes in constructed wetland for wastewater treatment[J]. Environmental Pollution and Control,128(8):616-619

[3] Zhang-Honggang,Hong-Jianming.2006.Functions of plants of constructed wetlands[J], Wetland Science,4(2):146-153

[4] Xu-Weiwei,Zhang-Beiping,Xiao Bo,Wang Jin,Guo Yong.2005.The functions of plant in constructed wetland for wastewater treatment[J],Safety and Environmental Engineerin, 12(2):41-44

[5] Cheng Wei,Cheng Dan,Li Qiang.2005.The purification principle and application of aquatic vascular macrophytes[J]. Industrial Safety and Environmental Protection, 31(1):6-9

[6] C.C. Tanner. 2001.Growth and nutrient dynamics of soft-stem bulrush in constructed wetlands treating nutrient-rich wastewaters[J]. Wetlands Ecology and Management, 9:49-73

[7] Tang-Shirong.2006.Phytoremediation of polluted environment principles and methods[M].Beijing:Science press,77-79

[8] SAMAKE Moussa,Wu Qi-tang,Mo Ce-hui,MOREL Jean-Louis.2003.Plants grown on sewage sludge in South China and its relevance to sludge stabilization and metal removal[J].Journal of Environmental Sciences, 15(5):622-627 
[9]Deng-Futang,SunShi,Deng-Fushang,WuGuang,LiQiang,Ma-Lihong,ChenRucai.2005.Purification efficiency of several macrophytes on polluted inflow river of Dianchi Lake[J].Guizhou Environmental Protection Science And Technology, $3: 7-12$

[10] Yuan-Donghai,Gao-Shixiang,Ren-Quanjin, Yin-Daqiang,WangLiansheng.2004.Study on purified efficiency of phosphorus and nitrogen from domestic sewage by several macrophytes in vertical flow constructed wetlands[J].Journal of Soil and Water Conservation, 18(4):78-80

[11] A. Fey ,G. Benckiser ,J.C.G. Ottow.1999.Emissions of nitrous oxide from a constructed wetland using a groundfilter and macrophytes in waste-water purification of a dairy farm[J]. Biol Fertil Soils,29:354-359

[12] Zhao-Jiangang,Liu-Lina,Chen-Zhanghe.2006.Study on removal rate of pollutants and plant growth in subsurface and surface flow constructed wetlands.[J].Ecologic Science ,25(1):74 77

[13]Zhong-Yushu,Wang-Guosheng,TianMin,Song-Honghai,Yu-Changbin,ZhangShaobing,Xui-Yanji-e,Gao-Hongwu,Tian-Wenda,Zhang-Changnan,ZhangXiuling.2006. Study on purifying waste water from paper making industry by reed marsh ecological system[J].Liaoning Agricultural Sciences, (3):6-8

[14]Yuan-Donghai,Ren-Quanjin,Gao-Shixiang,ZhangHong,Yin-DaQiang,WangLiansheng.2004.Purification efficiency of several wetland macrophytes on COD and nitrogen removal from domestic sewage $[\mathrm{J}]$.Chinese Journal of Applied Ecology, 15(12):2337 2341

[15] Weng-Meiya,Liu Peng,Xu-Gendi,Cai-Miaozhen.2005.Research progress of the treatment of polluted water in the artificial humid soil area [J].Journal of Anhui Agricultural Sciences ,33(7):1251-1253

[16] Liu-Zilian,Shi-Yongsheng,Li Peng.2005.Application of Constructed Wetland in Wastewater Treatment[J].Yunnan Chemical Technology, 32(6):60-63

[17] Robert M. Seams.1995.The evolution of greenways as an adaptive urban landscape form[J]. Landscape and Urban Planning, 33 :65-80

[18] International Environmental Protection Administration.2003. Water and wastewater monitoring and analysis methods[M].Beijing: China Environmental Science Press

[19] Wang Yi.2005.Study on purification ability of hydrophytes to domestic sewage in city[D].Sichuan:Sichuan Agricultural University

[20] Yin Jun,Wen Yue,Zhou Qi.2007.Microbial characteristics of constructed wetlands[J]. Environmental Science and Technology, 30(1):108-110 
[21] Zhao Jian,Zhu Wei,Zhao-Lianfang.2007.Efficiency and mechanism of treating polluted river water with constructed wetland[J]. Journal of Lake Sciences, 19(1):32-38 